\title{
MOBILITY MANAGEMENT FOR PROVIDING QOS IN LOCAL AREA WIRELESS NETWORKS
}

\author{
J. Antonio García-Macías, Franck Rousseau, Gilles Berger-Sabbatel, \\ Leyla Toumi, Andrzej Duda \\ LSR-IMAG Laboratory, Grenoble, France
}

\{amacias, rousseau, gberger, toumi, duda\}@imag.fr

\section{INTRODUCTION}

The purpose of this paper is to present our current work on mobility support integrated with QoS mechanisms in a wireless local area network. Such an environment becomes increasingly important with the emergence of ubiquitous mobile devices connected via wireless LANs and the development of future wireless telecommunication services such as UMTS. They contribute to the emergence of new real-time multimedia applications such as voice and video, networked games, or cooperative work that require better quality of service than current Best Effort. Integration of mobility with QoS support is a difficult challenge because of specific radio channel characteristics and complexity of mobility management.

Our approach is based on QoS mechanisms at the IP layer-Differentiated Services (DiffServ) [2]. We extend them to a wireless LAN environment so that we can provide consistent end-to-end quality of service to mobile hosts. If we want to support QoS for mobile hosts, we need to rethink our approach to mobility management. As the DiffServ QoS management is done at the IP level, we have to manage mobility also at the IP level, so that both issues can be addressed in an integrated way. However, the traditional approach to mobility at the IP level-Mobile IP [5, 6] does not take into account QoS requirements. Moreover, it is unsuitable for integration with QoS mechanisms because of its high overhead: triangular routing, address translation, and complex interaction between agents [3].

In our approach, we limit the scope of mobility management to a local subnetwork of wireless cells-we think that coupling QoS with mobility management makes sense only in such a case. We use a micro-mobility scheme implemented in the IPv6 layer with fast hand-offs between adjacent cells. It is the 
right level to deal with local mobility, because mobility is essentially a routing problem. Micro-mobility avoids address translation, traffic tunneling, and accelerates hand-offs. Coupled with the QoS management, it contributes to the overall end-to-end performance. A mobile station may decide to hand-off to an adjacent cell based on the current channel characteristics (signal to noise ratio), performance conditions, or access control policies.

The rest of the paper presents our mobility management scheme. Then we discuss briefly its integration with QoS management and compare with related work. We terminate with some conclusions and further developments.

\section{MOBILITY MANAGEMENT FOR FAST HAND-OFFS}

Each cell of a wireless LAN is managed by an Access Router $(A R)$ that forwards packets between mobile hosts in a cell. Access Routers are interconnected via a wired network and linked to an Edge Router (ER). There can be some intermediate routers between Access Routers and the Edge Router. For any pair of adjacent cells there is a cross-over router at the intersection of the routes going from Access Routers to the Edge Router. Figure 1 presents the main elements of the architecture.

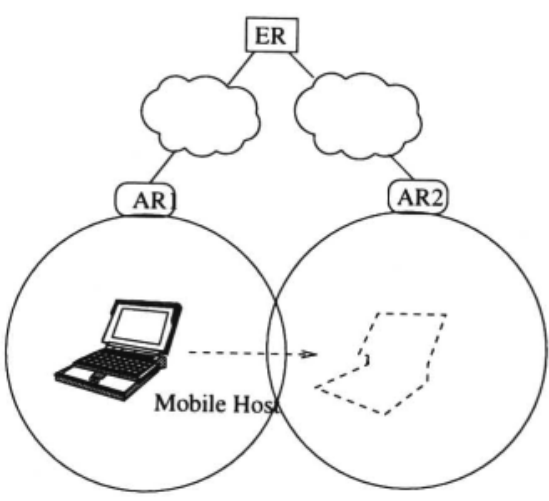

Figure 1. Architecture of mobility.

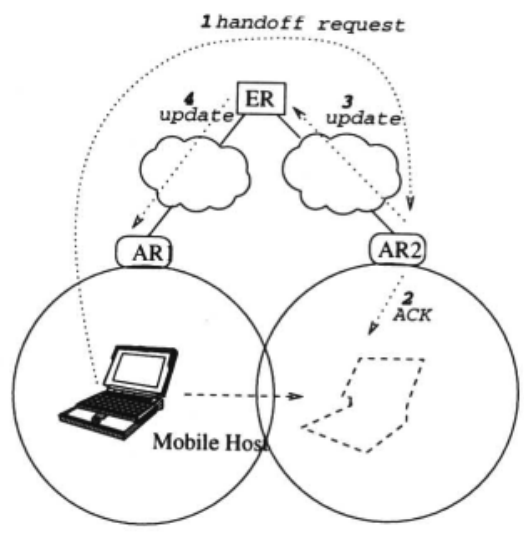

Figure 2. Mobility protocol.

As we have stated, one of the design requirements for our mobility management scheme was its integration with QoS support. Fast hand-offs can only be achieved when a mobile station keeps its IP address when moving to another cell, routes in the wired backbone being updated to reflect the new location of the station. Careful preparation of the new route in advance makes it possible to avoid lost packets and reduces the hand-off delay. We also assume that 
neighbour cells are overlapping so that the radio channels have sufficient quality during the hand-off.

We describe below the operation of our mobility management protocol during a hand-off (cf. Figure 2).

- Hand-off initiation. At some instant the mobile host decides to move to another cell. This decision can be based on some standard parameters such as the signal to noise ratio or it can take into account QoS parameters: the load or the number of stations in the current and in the adjacent cell (an increased number of stations means an increasing number of collisions at the MAC level). When the decision is taken, the mobile host sends a hand-off request to the target Access Router (AR2) via its current Access Router (AR1) to setup a new route (step 1). The request contains the address of the Access Router of the target cell and the current demand for bandwidth allocation.

- Hand-off request propagation. The current Access Router (AR1) propagates the hand-off request to the target Access Router (AR2) that checks whether the request can be satisfied or not. For example, if there are not enough resources, the hand-off may be denied. To avoid such a situation, which may severely affect QoS performance, Access Routers can pre-reserve resources in adjacent cells.

- Hand-off granted. If the hand-off request is accepted, the target router modifies its routing table by inserting a host route for the mobile host. The request is acknowledged to the mobile host via the wireless link of the target cell (step 2). The mobile host changes its routing table by specifying the target Access Router (AR2) as its default router. At this instant, the mobile host is able to communicate with mobiles in the target cell.

- New route setup. After sending the acknowledgment to the mobile host, the target Access Router (AR2) relays the hand-off request to all routers in the wired backbone up to the cross-over router (step 3). All routers update their routing tables by inserting a host route that goes via the target Access Router (AR2) to reflect the new location of the mobile host. At this instant, the traffic from hosts behind the Edge Router can be forwarded to the target cell using the new route.

- Old route deletion. The cross-over router forwards the hand-off request to all routers on the old route to the previous Access Router (step 4). The routers changes the old route in the routing tables. At this instant, the traffic from the previous cell can be forwarded to the target cell using the new route. 
In our scheme, we initiate a hand-off by contacting the current Access Router before using any resource of the target cell. The mobile host changes its routes and start using the target cell after the target Access Router has granted permission. This means that there are enough resources to satisfy the QoS requirements of the mobile host. The order of route updates prevents transient routing loops or the creation of multiple traffic streams during hand-off. Moreover, the scheme is optimized so that the traffic can be delivered as soon as possible to the new location: after the first route setup at the target Access Router (AR2), some part of the traffic to the mobile host can be already delivered; after step 3 and 4, the rest of the traffic is rerouted to the new location. Note also that some part of the operation is performed in parallel: hand-off propagation in the wired network and the movement of the mobile towards the target cell.

\section{QOS MANAGEMENT}

Our QoS architecture aims at extending DiffServ to a wireless LAN so that mobile hosts can benefit from different performance classes in a similar way to wired networks. We assume that the wired backbone is an over-provisioned LAN such as for example a switched Ethernet, so that the only performance critical parts of the global network are wireless links. All mobile hosts and Access Routers are provided with the DiffServ mechanisms to control traffic sources in function of varying conditions of a cell: parameters of traffic shapers and bandwidth allocations for QoS classes can be adjusted to provide requested performance behavior.

A 802.11 WLAN environment has specific characteristics that make it difficult to provide adequate quality of service: the radio channel is shared between all stations and the access overhead increases with the number of stations. Our approach to providing quality of service in a 802.11 environment is based on the following constraints:

- we limit the area of a cell so that all stations use the same high bit rate of the radio channel,

- we constraint traffic sources by configuring traffic shapers in stations to obtain desired $\mathrm{QoS}$ effects,

- we limit the number of stations allowed to use a cell (a hand-off request can be denied if there is no enough available bandwidth in a cell).

An Access Router manages QoS allocations in a cell: a mobile host informs it about the required bandwidth and the Access Router configures the QoS mechanisms of the mobile host. More details on the QoS management are given elsewhere [4]. 


\section{RELATED WORK}

Our mobility management scheme is similar to those studied in the HAWAII project [7]. HAWAII proposes four schemes: MSF, SSF, UNF, and MNF. In MSF, hand-off is initiated via the old base station and results in transient loops, whereas SSF requires more descriptive routing tables. UNF and MNF rely on the capacity of the mobile host to communicate with both base stations: the old and the new one. When a mobile host hand-offs into a new cell, routing tables in routers involved in the movement are modified starting from the new base station. The HAWAII mobility schemes have been only validated by simulation and they do not provide any specific QoS support. At the beginning, we considered the UNF scheme, however it does not take into account the QoS management-before using a cell, the new Access Router has to check whether the QoS requirements of the mobile host can be satisfied or not. So, in our mobility scheme, the hand-off request is sent first to the old Access Router and relayed to the new one.

\section{CONCLUSIONS AND FUTURE WORK}

We have implemented the basic elements of our architecture: the micromobility scheme and the intra-cell QoS management based on DiffServ. We use FreeBSD PC boxes that act as Access Routers to provide all required functions to mobile hosts. They are connected using wireless $11 \mathrm{Mb} / \mathrm{s} 802.11 \mathrm{~b}$ and 10 $\mathrm{Mb} / \mathrm{s}$ wired Ethernet cards. Access Routers and mobile hosts use an IPv6 stack developed in a French Next Generation Internet project [1].

We have measured performance of differentiation between two traffic sources: an UDP source generating a priority EF traffic and a TCP source generating an elastic BE traffic. Configuring the DiffServ mechanisms in the mobile host allows to limit the BE traffic to a given value $(2.4 \mathrm{Mb} / \mathrm{s})$ so that the delay of the EF class is only slightly disturbed by the BE class. In the case when mobile host does not require configuration of QoS mechanisms, the hand-off has taken around $5 \mathrm{~ms}$.

We are working on the integration of mobility management with QoS mechanisms: the in-band signaling protocol and the coupling of intra-cell QoS management with the management at the inter-cell level. This will allow dynamic changes in QoS allocations that adapt to varying conditions in the network. After the integration of the mechanisms, we will be able to provide more results on the performance of DiffServ classes during a hand-off. 


\section{Acknowledgments}

This work has been supported by the French Ministry of Industry National Network of Telecommunication Research via the AIRS project: "Integrated Architecture for Networks and Services".

\section{References}

[1] AIRS (2001). Integrated Architecture for Networks and Services. http://www-rp.lip6.fr/airs/.

[2] Blake et al., S. (1998). An Architecture for Differentiated Services. Internet RFC 2475.

[3] Chalmers et al., D. (1999). A Survey of Quality of Service in Mobile Computing Environments. IEEE Online communication Surveys, 2(2).

[4] García-Macías, J., Rousseau, F, Berger-Sabbatel, G., Leyla, T., and Duda, A. (2001). Quality of Service and Mobility for the Wireless Internet. In submitted for publication.

[5] Perkins, C. (1996). Mobile IP Specification. Internet RFC 2002.

[6] Perkins, C. and Johnson, D. (1996). Mobility Support in IPv6. In Mobile Computing and Networking, pages 27-37.

[7] Ramjee et al., R. (1999). HAWAII: A Domain-based Approach for Supporting Mobility in Wide-area Wireless Networks. In IEEE Int'l. Conf. Network Protocols. 